\title{
Unusual presentations of tuberculosis
}

\author{
B. T. LE ROUX
}

The Thoracic Unit, Wentworth Hospital, Durban, and the Department of Surgery, University of Natal

Four unusual examples of pulmonary and mediastinal tuberculosis are illustrated. Pulmonary lesions, and mediastinal tuberculosis, may mimic those lesions together grouped as 'mediastinal cysts and tumours'.

Pulmonary, mediastinal, glandular, and spinal tuberculosis remain a scourge and an important clinical problem in many parts of the world where public health facilities do not match up with the requirements of a large necessitous population. Among the Bantu and Asiatic (a remarkably large number of whom are diabetic) populations of the Province of Natal in South Africa, tuberculosis is common and must always be borne in mind as the cause of a pulmonary or mediastinal lesion. Four unusual examples of tuberculosis in juvenile patients, in only one of whom was there a diagnostic clue, are presented. That it is not always possible to decide on radiographic grounds that a lesion is mediastinal and not pulmonary is made clear from three of these cases, and a fourth example of difficulty in deciding where a lesion lies is illustrated.

\section{CASE HISTORIES}

CASE 1 An Asiatic girl of 8 years was admitted for investigation because a well-defined right apical radiographic opacity had been recognized when chest radiography was prompted by continued respiratory symptoms after a 'flu-like illness. By the time admission was arranged the child was symptom-free. Clarity of definition of the lower margin of the opacity (Fig. 1) suggested mediastinal neural tumour as a possible diagnosis. Two days after admission, before any investigation had been undertaken, the child coughed up about $50 \mathrm{ml}$ of blood-stained, purulent sputum. In this tubercle bacilli were recognized. A chest radiograph (Fig. 2) made three days after the first (Fig. 1) shows a right upper pulmonary cavity. The postero-anterior chest radiograph (Fig. 3) was made after 10 days of anti-tuberculosis chemotherapy.

CASE 2 A Bantu boy aged 6 years was admitted for investigation of a 'mediastinal' radiographic opacity. Chest radiography was prompted by loss of weight and cough. The posterior situation of the opacity (Fig. 4) made a thymic lesion unlikely.

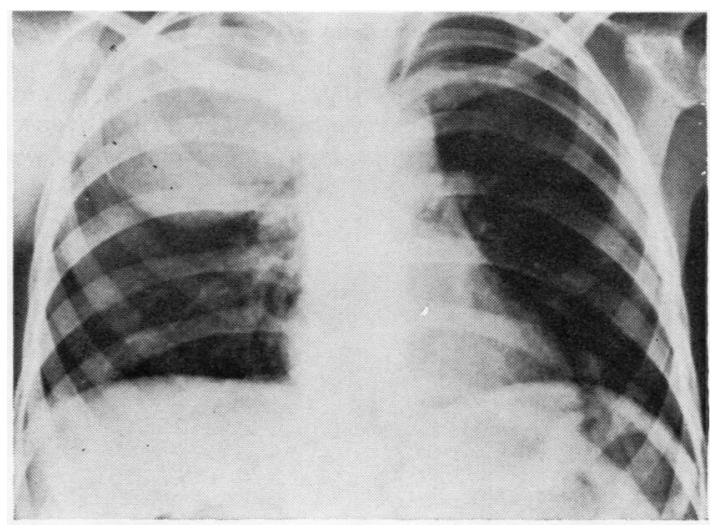

(a)

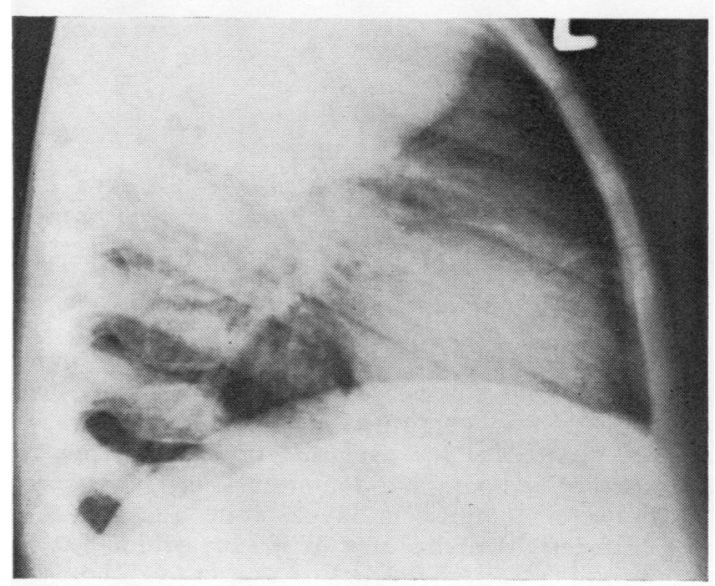

(b)

FIG. 1. Case 1. (a) Postero-anterior and (b) right lateral radiographs. Mediastinal neural tumour was the diagnosis most favoured on radiographic grounds. 


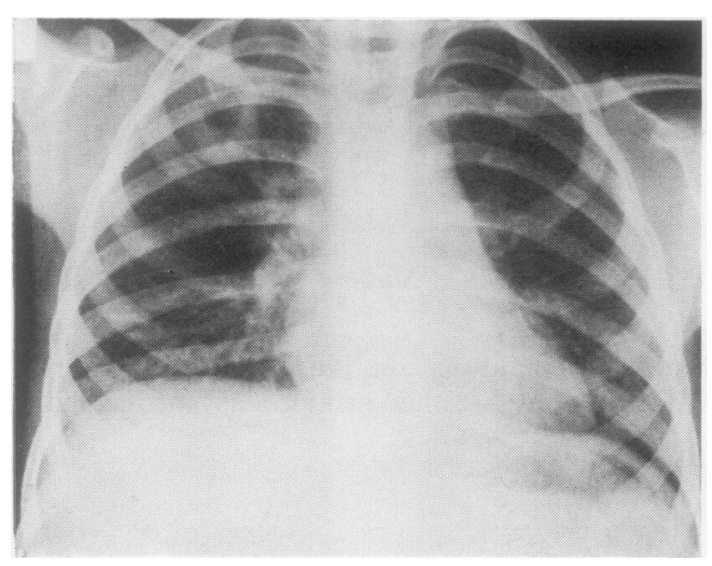

FIG. 2. Case 1. Postero-anterior radiograph three days after that shown in Fig. 1 and after the patient had coughed up a quantity of bloodstained pus. The right upper pulmonary cavity is clear.

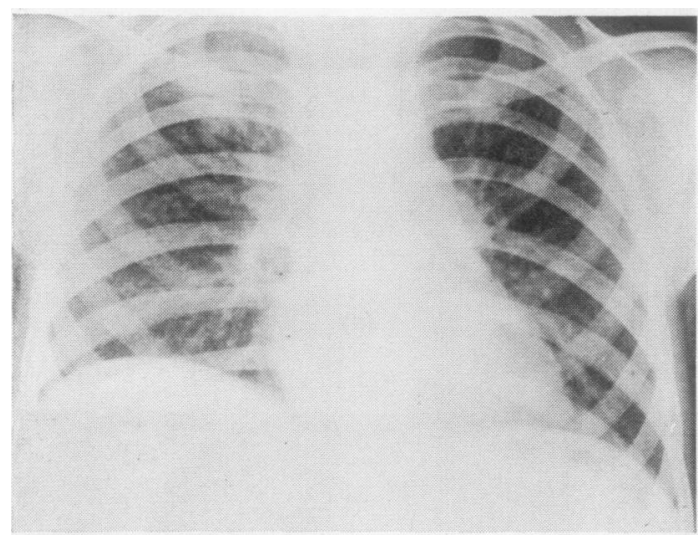

FIG. 3. Case 1. Chest radiograph after 10 days' antituberculosis chemotherapy. The cavity is no longer visible. Management thereafter was in the hands of experts in tuberculosis.

Vertebrae in relation to the opacity were 'motheaten,' and cold abscess seemed the most likely diagnosis. This was confirmed by aspiration. Air was allowed to replace the aspirated tuberculous pus for purposes of radiographic definition (Fig. 5). Tubercle bacilli were identified in the aspirate and the child was transferred to the care of a sanatorium.

CASE 3 A Bantu boy aged 17 years was admitted for investigation of a radiographic abnormality recognized when he was submitted to routine surveillance on taking up employment (Fig. 6). Clinical examination was unhelpful. On radiographic grounds foregut duplication seemed a likely diagnosis. Preliminary

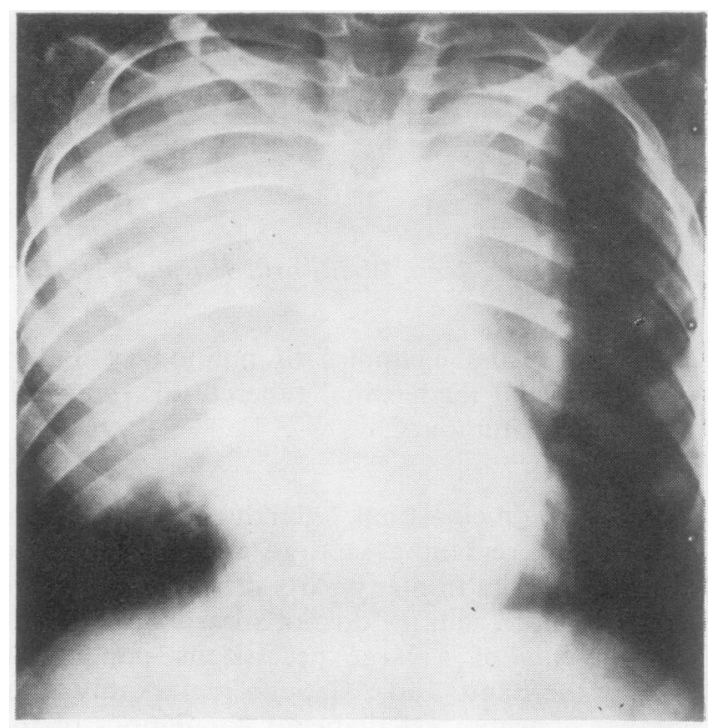

(a)

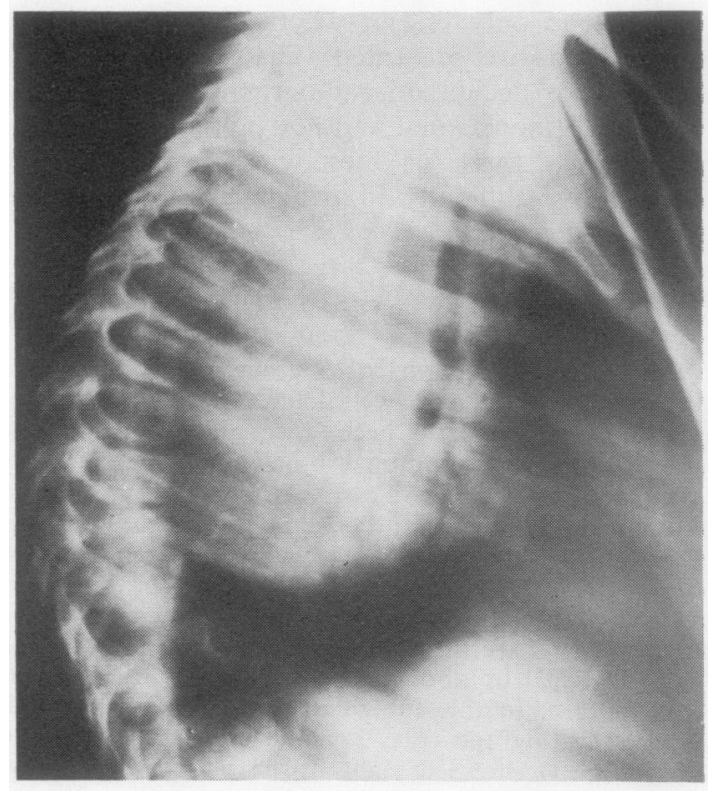

(b)

FIG. 4. Case 2. (a) Postero-anterior and (b) right lateral films. Although the child was admitted for investigation of a mediastinal lesion, its posterior situation made a thymic lesion unlikely, and the 'moth-eaten' appearance of the upper thoracic vertebrae strongly suggested the diagnosis of cold abscess. 


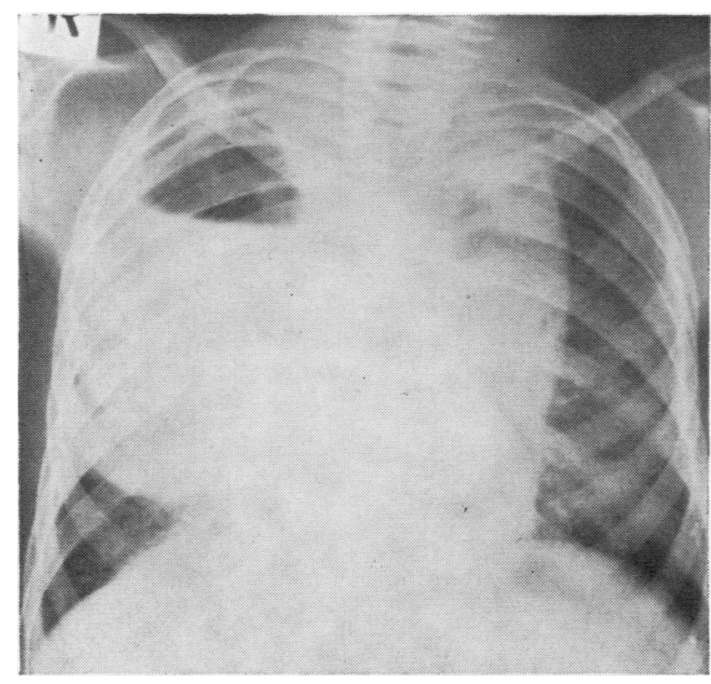

FIG. 5. Case 2. Postero-anterior chest radiograph made after aspiration of the cold abscess.

investigations were unrewarding. At exploratory thoracotomy a single large gland was enucleated from the mediastinum in relation to the trachea and from behind the azygos vein. The gland was shown to be tuberculous.

CASE 4 This is an almost exact replica of case 3. The lateral films available are of such poor quality that only the relevant postero-anterior film (Fig. 7) is shown. Management was similar.

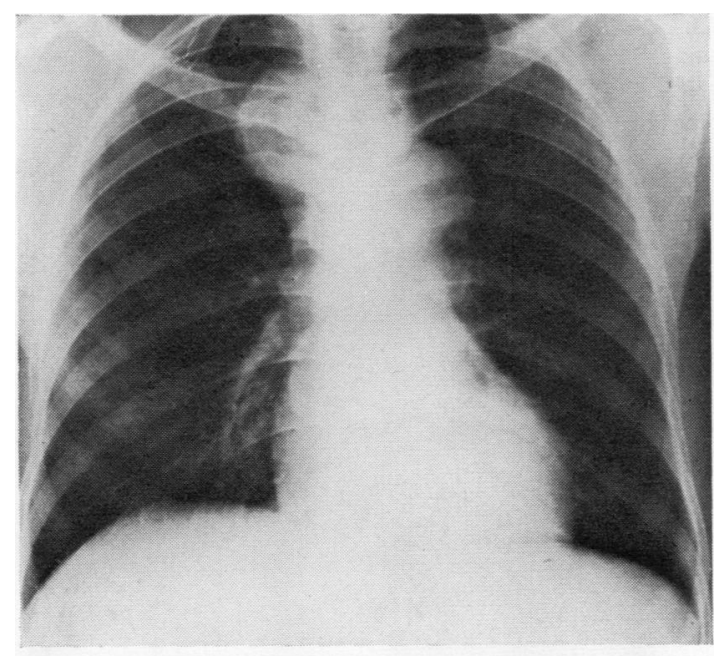

(6a)

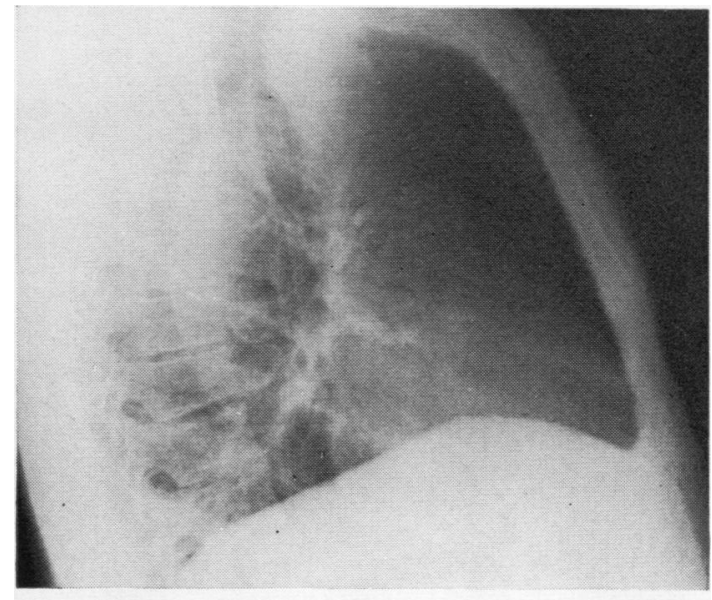

(6b)

FIG. 6. Case 3. (a) Postero-anterior and (b) right lateral radiographs showing a large tuberculous mediastinal gland, interpreted preoperatively as most likely to be a foregut duplication.

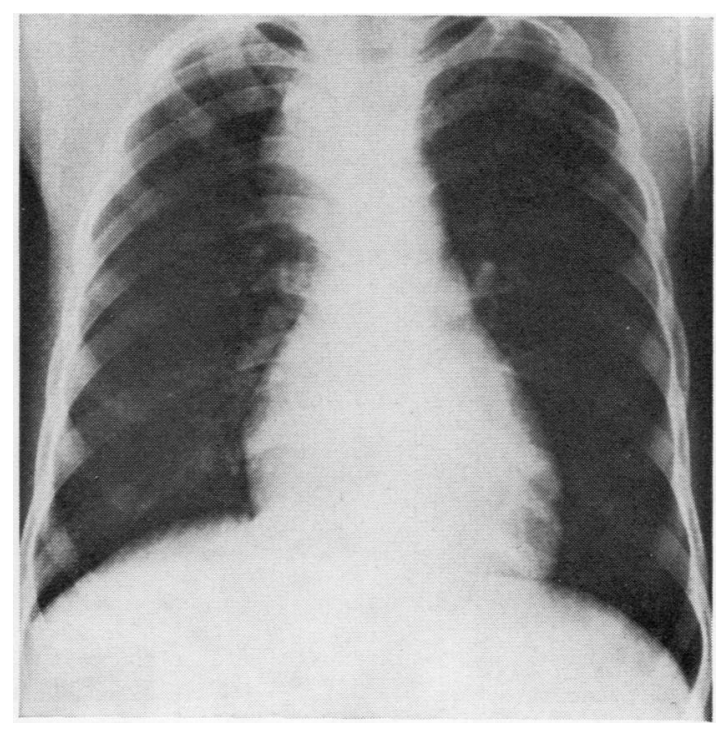

FIG. 7. Case 4. Postero-anterior radiograph similar to that shown in Fig. 6. The pre-operative diagnosis from this patient's radiograph was also foregut duplication. The lesion was a single large mediastinal tuberculous gland. 


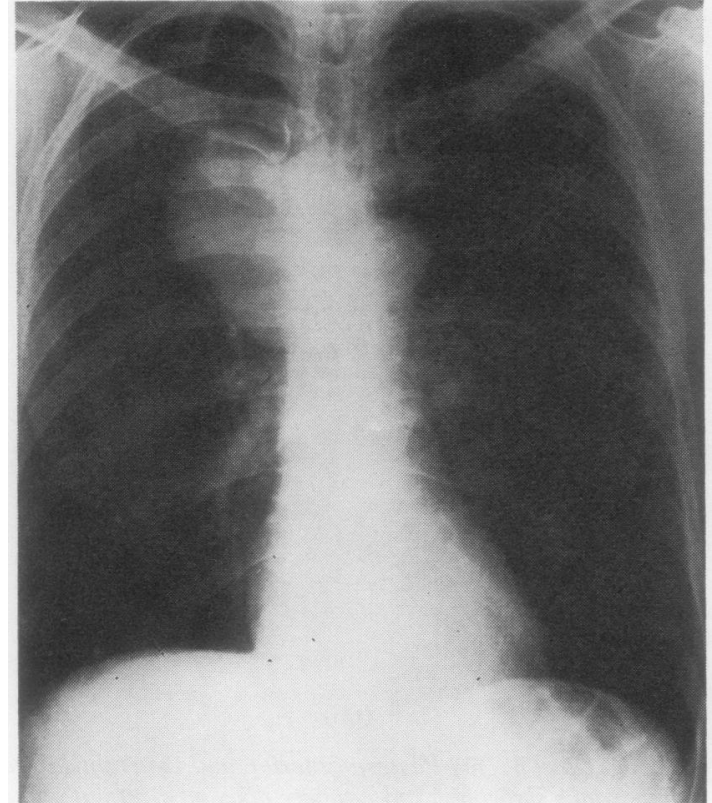

(a)

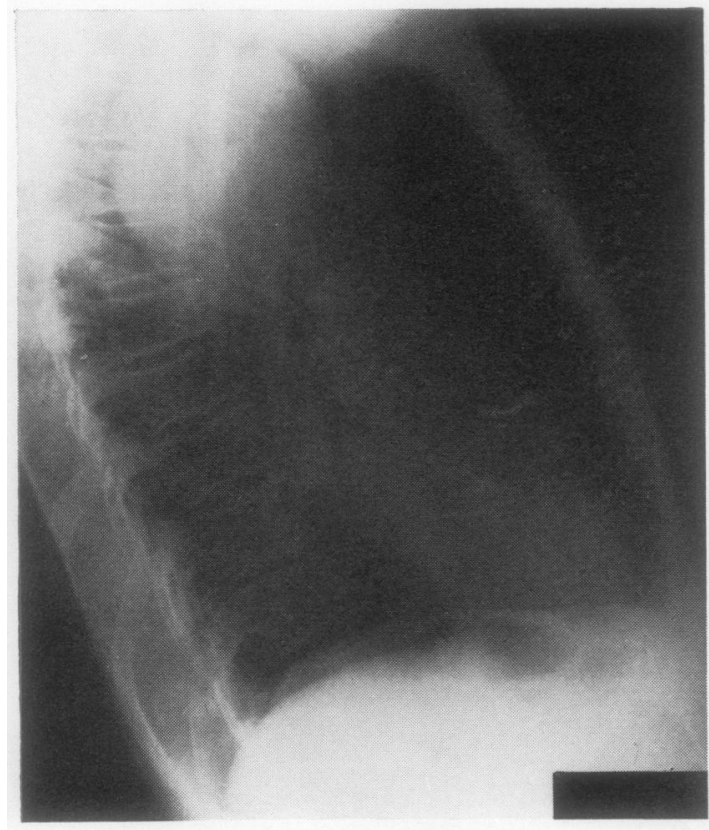

(b)

FIG. 8. Case 5. (a) Postero-anterior and (b) right lateral radiographs. The lesion was thought to be most likely mediastinal rather than pulmonary.

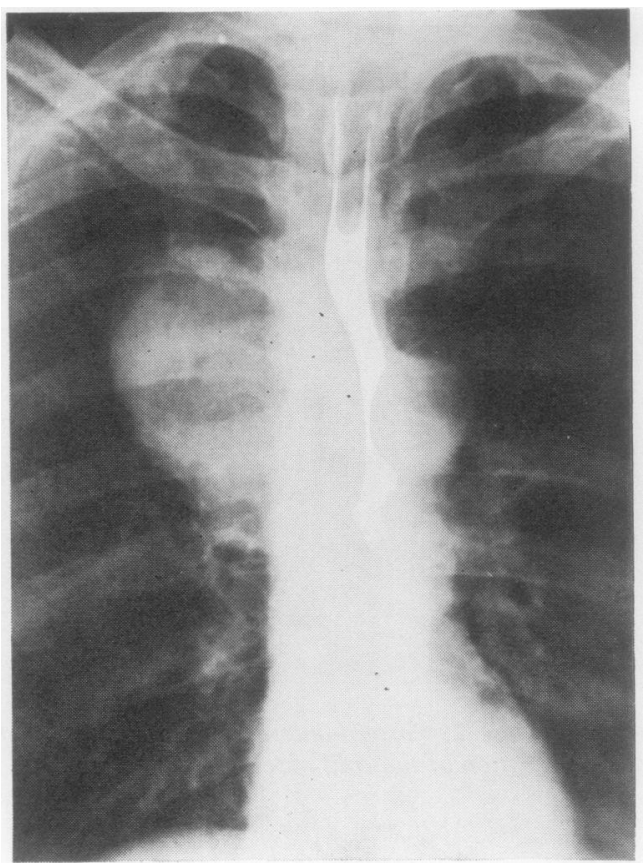

FIG. 9. Case 5. Displacement of the barium-filled oesophagus is shown.

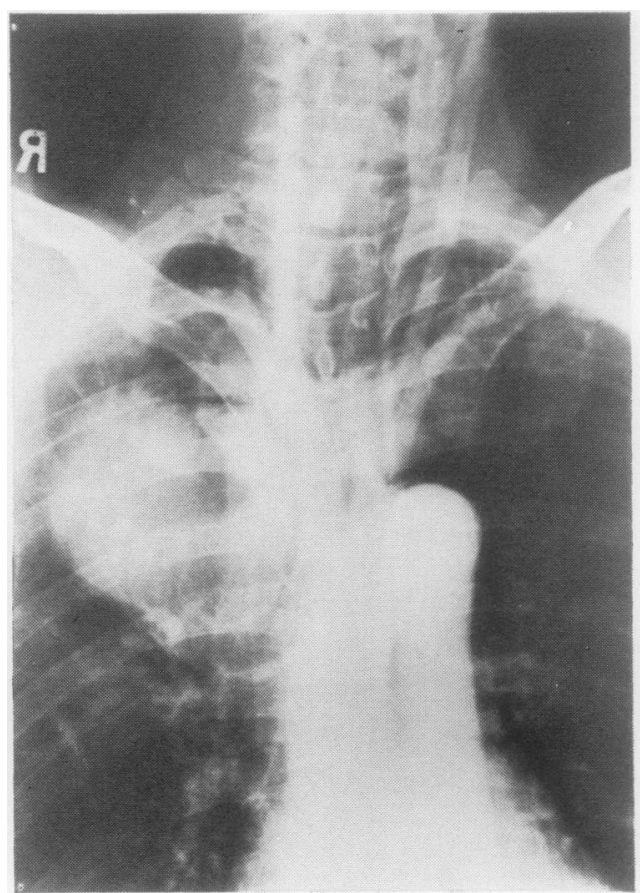

FIG. 10. Case 5. An aortogram which is normal. 
CASE 5 An adult Caucasian of 56 years was admitted for investigation when a right-sided radiographic abnormality was recognized in relation to a respiratory illness of short duration. The opacity (Fig. 8) abutted on the mediastinal shadow and was clearly defined and lobulated. Clinical investigation was unhelpful. A penetrated view, with barium, showed the oesophagus to be a little displaced (Fig. 9). An aortogram was normal (Fig. 10). All who had the opportunity of scrutinizing the available radiographic information agreed that the lesion was mediastinal and not pulmonary. At exploratory right thoracotomy a tumour in the right upper lobe was managed by lobectomy. Histologically the tumour was shown to be a carcinoid type of bronchial adenoma without glandular metastasis. Recovery from thoracotomy was uneventful and the patient remains well.

\section{COMMENT}

Distinction between mediastinal and pulmonary lesions, on radiographic grounds alone, is often not possible. Glandular tuberculosis must be remembered as a cause of an isolated large mediastinal shadow. Cold abscess may achieve a very large size. It is standard practice, where there is doubt about the nature of a peripheral pulmonary opacity, to undertake exploratory thoracotomy after a preliminary period of antituberculosis chemotherapy. This routine should be extended to include the surgical management of lesions thought likely to be mediastinal rather than pulmonary. 